OPEN ACCESS

Edited by:

Christy S Carter,

University of Alabama at Birmingham,

United States

Reviewed by:

Sanjib Guha,

University of Rochester, United States

Kimberly Ann Greer,

Prairie View A\&M University,

United States

*Correspondence:

Lorena Sordo

Lorena.Sordo@ed.ac.uk

Specialty section:

This article was submitted to

Aging, Metabolism and Redox Biology,

a section of the journal

Frontiers in Aging

Received: 23 March 2021

Accepted: 27 May 2021

Published: 07 June 2021

Citation:

Sordo L, Martini AC, Houston EF, Head E and Gunn-Moore D (2021) Neuropathology of Aging in Cats and

its Similarities to Human

Alzheimer's Disease.

Front. Aging 2:684607.

doi: 10.3389/fragi.2021.684607

\section{Neuropathology of Aging in Cats and its Similarities to Human Alzheimer's Disease}

\author{
Lorena Sordo $^{1 *}$, Alessandra C. Martini ${ }^{2}$, E. Fiona Houston ${ }^{1}$, Elizabeth $\mathrm{Head}^{2}$ and \\ Danièlle Gunn-Moore ${ }^{1}$
}

${ }^{1}$ The Royal (Dick) School of Veterinary Studies and The Roslin Institute, The University of Edinburgh, Edinburgh, United Kingdom, ${ }^{2}$ Department of Pathology and Laboratory Medicine, University of California, Irvine, Irvine, CA, United States

Elderly cats develop age-related behavioral and neuropathological changes that ultimately lead to cognitive dysfunction syndrome (CDS). These neuropathologies share similarities to those seen in the brains of humans with Alzheimer's disease (AD), including the extracellular accumulation of $\beta$-amyloid $(A \beta)$ and intraneuronal deposits of hyperphosphorylated tau, which are considered to be the two major hallmarks of AD. The present study assessed the presence and distribution of $A \beta$ and tau hyperphosphorylation within the cat brain ( $n=55$ cats), and how the distribution of these proteins changes with age and the presence of CDS. For this, immunohistochemistry was performed on seven brain regions from cats of various ages, with and without CDS $(n=10$ with CDS). Cats accumulate both intracytoplasmic and extracellular deposits of $A \beta$, as well as intranuclear and intracytoplasmic hyperphosphorylated tau deposits. Large extracellular aggregates of $A \beta$ were found in elderly cats, mainly in the cortical brain areas, with occasional hippocampal aggregates. This may suggest that these aggregates start in cortical areas and later progress to the hippocampus. While $A \beta$ senile plaques in people with $A D$ have a dense core, extracellular $A \beta$ deposits in cats exhibited a diffuse pattern, similar to the early stages of plaque pathogenesis. Intraneuronal $A \beta$ deposits were also observed, occurring predominantly in cortical brain regions of younger cats, while older cats had few to no intraneuronal $A \beta$ deposits, especially when extracellular aggregates were abundant. Intracytoplasmic hyperphosphorylated tau was found within neurons in the brains of elderly cats, particularly in those with CDS. Due to their ultrastructural features, these deposits are considered to be pre-tangles, which are an early stage of the neurofibrillary tangles seen in $A D$. The largest numbers of pre-tangles are found mainly in the cerebral cortex of elderly cats, whereas lower numbers were found in other regions (i.e., entorhinal cortex and hippocampus). For the first time, intranuclear tau was found in both phosphorylated and non-phosphorylated states within neurons in the cat brain. The highest numbers of intranuclear deposits were found in the cortex of younger cats, and this tended to decrease with age. In contrast, elderly cats with pre-tangles had only occasional or no nuclear labelling.

Keywords: neuropathology, aging, Alzheimer's disease, cats, model, b-amyloid, tau, CDS 


\section{INTRODUCTION}

In humans, age-related brain changes, such as brain atrophy, neuronal loss, and vascular pathology, may ultimately lead to dementia and Alzheimer's disease (AD) (Raz, 2004; Jack et al., 2005; Glabe, 2006). The abnormal deposition of senile plaques containing $\beta$-amyloid $(\mathrm{A} \beta)$ and neurofibrillary tangles (NFT) composed of hyperphosphorylated tau are major pathognomonic neuropathologies of $\mathrm{AD}$, and are thought to play a key role in its development (Hardy and Allsop, 1991; Thal et al., 2002; Perl, 2010).

Similarly, aged cats develop age-related cognitive decline and eventually dementia, which is known as cognitive dysfunction syndrome (CDS; feline dementia) (Gunn-Moore et al., 2007; Landsberg et al., 2010). While the neuropathology of this condition is still poorly understood, several age-related neurodegenerative changes (i.e., brain atrophy, neuronal loss, vascular changes, and abnormal protein deposition) are found in the brains of elderly cats (Head et al., 2005; Gunn-Moore et al., 2006).

$\beta$-amyloid is generated from the cleavage of the amyloid precursor protein (APP) by $\beta$ and $\gamma$-secretases. Since cleavage occurs at different sites, various lengths of $A \beta$ can be formed, with $A \beta 40$ and $A \beta 42$ being found most commonly in the brain of patients with $\mathrm{AD}$. While $\mathrm{A} \beta 40$ is the most abundant and tends to accumulate within blood vessels, $A \beta 42$ tends to accumulate in the extracellular space and form the characteristic senile plaques of AD (Hardy and Allsop, 1991; Perl, 2010).

Diffuse $A \beta$ aggregates are found in the brains of cats over 10 years of age (Cummings et al., 1996; Nakamura et al., 1996; Brellou et al., 2005; Head et al., 2005; Gunn-Moore et al., 2006; Gunn-Moore et al., 2007). Since these deposits do not have dense cores and share similarities to the diffuse deposits seen in nondemented humans, it has been suggested that the $A \beta$ deposits seen in cats are less mature than the characteristic senile plaques of AD (Cummings et al., 1996; Nakamura et al., 1996; Colle et al., 2000; Head et al., 2000; Gunn-Moore et al., 2007). Similarly to human $A \beta$, deposits in cats are predominantly formed from the A $\beta 42$ peptide (Cummings et al., 1996; Head et al., 2005). The lack of $A \beta 40$ in cats may be due to its higher solubility, which promotes its rapid clearance, impeding its accumulation within the brain (Hyman et al., 1993; Cummings et al., 1996; Wisniewski et al., 1996). However, the A $\beta 40$ peptide is found in association with cerebral blood vessels walls in cats, termed cerebral amyloid angiopathy (Nakamura et al., 1996). Both the pattern and distribution of $\mathrm{A} \beta$ in cats are more similar to those found in the elderly, non-demented human brain rather than patients with AD (Cummings et al., 1996; Nakamura et al., 1996; Brellou et al., 2005; Head et al., 2005; Gunn-Moore et al., 2007).

Tau is a microtubule-associated protein located in the cytosol of the cells and provides microtubule stability (Lee et al., 2005; Kametani and Hasegawa, 2018). When abnormally hyperphosphorylated, tau aggregates into paired helical filaments (PHF) that subsequently form NFT (Goedert et al., 1989; Hardy and Allsop, 1991; Lee et al., 2005; Perl, 2010).

Cats produce similar tau isoforms to those seen in humans (Chambers et al., 2015); however, there is still controversy regarding the presence of NFT in the brains of elderly cats. While some research found no evidence of NFT (Nakamura et al., 1996;
Kuroki et al., 1997), others have reported intracytoplasmic hyperphosphorylated immunolabelling within neurons, which is believed to be an early stage of NFT, known as pre-tangles (Head et al., 2005; Gunn-Moore et al., 2006; Chambers et al., 2015).

The aim of this study was to assess the presence and distribution of $A \beta$ and tau pathology in the brains of cats of various ages, with and without CDS. It was hypothesized that elderly cats would have larger and more frequent extracellular $\mathrm{A} \beta$ deposits and higher numbers of pre-tangles than younger cats. It was also hypothesized that these changes would be more evident in cats with CDS.

\section{MATERIALS AND METHODS}

\section{Sample Collection}

The brains of cats of different ages were collected post-mortem through the routine necropsy service at The Royal (Dick) School of Veterinary Studies (RDSVS). Some of these cats were patients at the RDSVS Hospital for Small Animals, while others came from a rescue center in Scotland. Most of the cats were affected by old age and/or chronic disease and were euthanized after veterinary assessments revealed that further treatment would not benefit quality of life. A small number of cats were euthanized after being diagnosed with behavioral problems (e.g., untreatable aggression) that would impede their rehoming. In all cases, the owners or shelter manager gave written consent to donate the cats' bodies for research purposes.

Ethical approval was obtained through the Veterinary Ethical Review Committee from the RDSVS, The University of Edinburgh (VERC 50.17 and 30.20).

Some of the patients had been diagnosed with CDS before euthanasia. For this diagnosis to be made, cats had to have displayed at least one behavioral change associated with CDS for at least three months (Gunn-Moore et al., 2007; Landsberg et al., 2010; Sordo and Gunn-Moore, 2021), and the behavioral changes could not be attributed to any other medical condition.

After collection, brains were fixed by immersion in $10 \%$ buffered formalin for no less than one week. After fixation, transversal sections of the brain were taken from the rostral cortex (Figure 1A), parietal cortex, including hippocampus (Figure 1B), occipital cortex, including the entorhinal cortex (Figure 1C), the locus coeruleus and the cerebellum (Figure 1D).

Sections were processed overnight in paraffin (Leica Tissue Processor ASP300S; Leica Biosystems ${ }^{\mathrm{TM}}$ ) and blocks were made. Consecutive slices of $6 \mu \mathrm{m}$ were taken from the blocks using a microtome (Microtome HM325; Leica Biosystems ${ }^{\mathrm{TM}}$ ) and mounted on positively charged glass slides $\left(\right.$ Polysine $\left.{ }^{\circledR}, \mathrm{VWR}^{\mathrm{TM}}\right)$.

\section{Immunohistochemistry}

Consecutive $6 \mu \mathrm{m}$ sections were taken from the rostral, parietal (including hippocampus), and occipital (including entorhinal cortex) lobes, plus of the cerebellum and locus coeruleus. These regions are those that are typically affected by $A \beta$ and hyperphosphorylated tau in the AD brain. After deparaffinization and rehydrating sections, antigen retrieval pre-treatment was performed by immersing slides in $90 \%$ formic acid for $6 \mathrm{~h}$ 


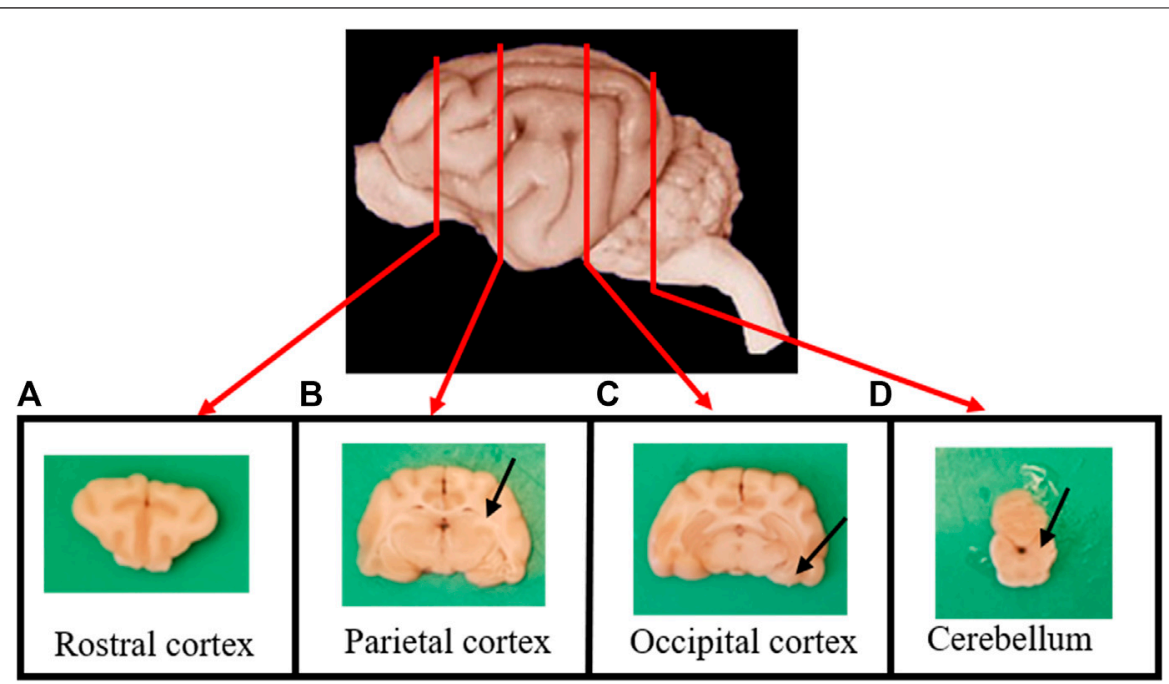

FIGURE 1 | Transverse sections of the brain were taken from (A) the rostral cortex; (B) the parietal cortex, including the hippocampus (arrow); (C) the occipital cortex, including the entorhinal cortex (arrow); and (D) the cerebellum and the locus coeruleus (arrow).

TABLE 1 | Primary antibodies for the detection of $B$-amyloid and tau proteins in the cat brain.

\begin{tabular}{lc}
\multicolumn{1}{c}{ Clone } & Dilution \\
\hline 4G8 & $1: 1,500$ \\
mOC64 & $1: 6,000$ \\
8E6/C11 & $1: 1,000$ \\
1E1/A6 & $1: 1,000$ \\
Tau46 & $1: 1,000$ \\
AT8 & $1: 200$ \\
A15091A & $1: 1,000$ \\
AT100 & $1: 1,000$
\end{tabular}

(Kraszpulski et al., 1998) for antibody 4G8, and heat incubation $\left(120^{\circ} \mathrm{C}\right.$ for $\left.40 \mathrm{~min}\right)$ in sodium citrate buffer $(10 \mathrm{mM}, \mathrm{pH}$ 6) for all other antibodies. All antibodies were incubated in $3 \%$ hydrogen peroxide in $10 \%$ methanol for $30 \mathrm{~min}$ to block endogenous peroxidase activity. Sections were incubated overnight at room temperature in each of the primary antibodies: two anti- $\beta$-amyloid and six anti-tau antibodies, listed in Table 1. Bound antibody was detected by incubating slides in biotinylated goat anti-mouse/rabbit antibodies (1:500; ThermoFisher Scientific ${ }^{\mathrm{TM}}$ ) for one hour, followed by incubation in $\mathrm{ABC}$ peroxidase kit (1:100; ThermoFisher Scientific $^{\mathrm{TM}}$ ) for $30 \mathrm{~min}$. Slides were visualized with $3,3^{\prime}$ diaminobenzidine substrate (1:100; DAB; Sigma-Aldrich ${ }^{\mathrm{TM}}$ ) under light microscope (Nikon ${ }^{\mathrm{TM}}$ Brightfield). Negative controls were used by omitting the primary antibodies. Immunostaining was conducted while blind to the groupings of the animals.

\section{Validation of Intranuclear Hyperphosphorylated tau Immunolabelling Isotype Controls}

To validate that intranuclear immunolabelling was not caused by artifact or non-specific staining, IHC was performed following the above protocol and using antibodies with the same isotypes for AT8 (mouse IgG isotype control; ThermoFisher Scientific ${ }^{\mathrm{TM}}$ ), AT100 (mouse IgG1 kappa isotype control; ThermoFisher Scientific ${ }^{\mathrm{TM}}$ ), and A15091A antibodies (mouse IgG2b kappa isotype control; Biolegend ${ }^{\mathrm{TM}}$ ) as negative controls.

\section{Dephosphorylation of the tau Protein}

To further validate that the intranuclear labelling observed in cat brains reflected the hyperphosphorylated state of the protein, as opposed to the non-phosphorylated protein, we used the approach of dephosphoryling the protein in brain samples. To achieve this, IHC was performed by following the protocol described above, for the same hyperphosphorylated tau antibodies (i.e., AT8, AT100, and A15091A), separately; however, to dephosphorylate the protein, slides were incubated in alkaline phosphatase (FastAP Thermosensitive Alkaline phosphatase; ThermoFisher Scientific ${ }^{\mathrm{TM}}$ ) following manufacturers' protocol at $37^{\circ} \mathrm{C}$ for one hour, followed by three $5 \mathrm{~min}$ washes in fresh bovine serum albumin, before adding the primary antibodies using the protocol described in Immunohistochemistry. 


\section{Protein Extraction}

Extracts from the cytoplasm, the cytoskeleton, the soluble nuclear fractions, and the chromatin bound nuclear fractions were obtained from frozen cat brain tissue (a 10-year-old cat) by using the Subcellular Protein Fractionation Kit for Tissues (ThermoFisher Scientific ${ }^{\mathrm{TM}}$ ) following manufacturer's protocol.

\section{Western Blot}

Extracts from the different fractions $(15 \mu \mathrm{l})$ obtained with the Subcellular Protein Fractionation Kit for Tissues (ThermoFisher Scientific $^{\mathrm{TM}}$ ) were prepared in a buffer containing NuPAGE ${ }^{\mathrm{TM}}$ LDS Sample Buffer (4x) and NuPAGE ${ }^{\mathrm{TM}}$ MES running buffer (10x) and were loaded into a 12 well NuPAGE ${ }^{\mathrm{TM}}$ pre-cast gel, $4-12 \%$ bis-tris. The gel ran at $50 \mathrm{~V}$ for $20 \mathrm{~min}$, followed by $120 \mathrm{~V}$ for $20 \mathrm{~min}$, and $200 \mathrm{~V}$ for $40 \mathrm{~min}$. The proteins were then transferred onto an Immobilon-FL PVDF membrane $\left(\right.$ Millipore $^{\mathrm{TM}}$ ) using a semi-dry electroblotting unit (TransBlot $^{\circledR}$ SD semi-dry transfer cell; Bio-Rad ${ }^{\mathrm{TM}}$ ), run at $0.06 \mathrm{~A}$ and $18 \mathrm{~V}$ for one hour. The PVDF membrane was transferred into LICOR blocking buffer (Licor Biosciences ${ }^{\mathrm{TM}}$ ) for one hour. Primary antibodies were added (AT8, 1:1,000; and Tau46, 1: $5,000)$, and blots were incubated at $4^{\circ} \mathrm{C}$ on a roller overnight. Goat anti-mouse antibodies were used as secondary antibodies (IRdye 680RD; Licor Biosciences $\left.{ }^{\mathrm{TM}}\right)$. Analysis was performed using LICOR odyssey (700 $\mathrm{nm}$ wavelength).

\section{Quantification of Protein Deposition}

Initially, all of the slides were visually inspected by light microscopy; this included the slides from the rostral, parietal, and occipital regions, plus the cerebellum, that had been obtained after performing IHC with all the eight antibodies.

For the automated quantification of protein deposition, only the slides stained against mOC64 for $\beta$-amyloid, and AT8 and AT100 for hyperphosphorylated tau, from all brain regions, were scanned using a Leica Aperio Versa 200 scanner (Leica Biosystems $^{\mathrm{TM}}$ ).

Images were transferred to the Aperio eSlide Manager ${ }^{\circledR}$ v12.4.0.5043 and visualized with the Aperio ImageScope ${ }^{\circledR}$ v12.3.3.5048. Annotation boxes of $600 \mu \mathrm{m}$ of width, height and length were manually placed for each brain region of interest; in total five annotation boxes were created for gray matter, and five for white matter; plus, three boxes for hippocampus, and three for cerebellum. The Positive Pixel Count v9 algorithm was used for the assessment of positive immunolabelling for the load and burden (see below) of $A \beta$ and hyperphosphorylated tau. In addition, the Nuclear Algorithm v9 was used for the assessment of positive immunolabelling for intranuclear hyperphosphorylated tau.

Data from the Positive Pixel Count v9 algorithms were exported to an excel file, where the load (i.e., percentage of total positive pixels) and burden (i.e., the proportion of the total area assessed that contains positive labelling) of both $\beta$ amyloid and hyperphosphorylated tau were calculated, by using the following equations:

$$
\text { Load of protein }(\%)=\left(\frac{N w p+N p+N s p}{N \text { total }}\right) \times 100
$$

$$
\text { Burden of protein }=\frac{\frac{N w p+N p+N s p}{\text { Total area }}}{10,000} \text { pixels } / \mathrm{mm}^{2}
$$

where,

Nwp = Number of weak positives.

$\mathrm{Np}=$ Number of (moderate) positives.

Nsp $=$ Number of strong positives.

Ntotal $=$ Number of total positives and negatives.

Total area was $1.8 \mathrm{~mm}^{2}$ for cortical regions and $1.08 \mathrm{~mm}^{2}$ for cerebellum and hippocampus. These area measures were calculated by multiplying two dimensions of the annotation boxes (i.e., $600 \mu \mathrm{m} \times 600 \mu \mathrm{m}$ ), multiplying the result by the number of annotation boxes created for each region (i.e., multiply by five for cortical regions and by three for hippocampus and cerebellum) and converting the result to $\mathrm{mm}^{2}$.

Data obtained from the Nuclear Algorithm v9, including the total percentage of nuclei stained and the degree of staining (i.e., $1+, 2+$, and $3+$ ) were exported to an excel sheet and used for statistical analyses.

\section{Data Analysis}

Data were analyzed using Minitab ${ }^{\circledR}$ v19.2020.1 statistical software for Windows.

Anderson-Darling tests were performed to assess normality. Non-normal data were transformed using Box-Cox transformation or Johnson transformation. Bartlett tests for equal variances were performed with $95 \%$ confidence level (CI), assuming normal distribution. Non-parametric tests were performed in the variables in which transformation failed.

To determine whether there were differences in the positive immunolabelling of proteins (mOC64 for $\beta$-amyloid, and AT8 and AT100 for hyperphosphorylated tau) in the different age groups, one-way ANOVA were performed assuming equal variances (if applicable) with two-sided 95\% CI and a significance level of $a=0.05$. Subsequently, we determined which group differences were significant using Tukey tests and Games-Howell post-hoc tests. Kruskal-Wallis tests were performed to assess differences in non-normal data.

To determine whether there were differences in the positive immunolabelling of the proteins between gray and white matter, 2 -sample $t$-tests were performed with a $95 \% \mathrm{CI}$ and a hypothesized difference of zero, assuming equal variances. Mann-Whitney tests were performed to assess differences in non-normal data.

To determine whether there were differences in the positive immunolabelling of the proteins between cats with and without CDS, 2-sample $t$-tests were performed as described above.

Finally, chi-square tests were performed to determine whether there was an association between cognitive status (i.e., cats with and without CDS) and the presence of pre-tangles.

\section{RESULTS}

\section{Demographics}

A total of 55 cat brains were collected at necropsy. The majority of cats were non-pedigree $(98.2 \% ; n=54)$, with only one cat being a 
TABLE 2 | Percentage of cats from the Prime and Super Senior groups showing intracytoplasmic and extracellular A $\beta$ deposits in the different brain regions, with both 4 G8 and mOC64 antibodies.

\begin{tabular}{|c|c|c|c|c|c|c|c|c|}
\hline & \multicolumn{4}{|c|}{ 4G8 antibody } & \multicolumn{4}{|c|}{ mOC64 antibody } \\
\hline & \multicolumn{2}{|c|}{ Intracellular $\mathbf{A} \boldsymbol{\beta}$} & \multicolumn{2}{|c|}{ Extracellular $\mathbf{A} \boldsymbol{\beta}$} & \multicolumn{2}{|c|}{ Intracellular $\mathbf{A} \boldsymbol{\beta}$} & \multicolumn{2}{|c|}{ Extracellular $\mathbf{A} \boldsymbol{\beta}$} \\
\hline & Prime & $\begin{array}{l}\text { Super } \\
\text { senior }\end{array}$ & Prime & $\begin{array}{l}\text { Super } \\
\text { senior }\end{array}$ & Prime & $\begin{array}{l}\text { Super } \\
\text { senior }\end{array}$ & Prime & $\begin{array}{l}\text { Super } \\
\text { senior }\end{array}$ \\
\hline Rostral cortex & $83 \%$ & $77 \%$ & $50 \%$ & $62 \%$ & $100 \%$ & $46 \%$ & $50 \%$ & $77 \%$ \\
\hline Parietal cortex & $100 \%$ & $85 \%$ & $33 \%$ & $54 \%$ & $50 \%$ & $54 \%$ & $67 \%$ & $85 \%$ \\
\hline Occipital cortex & $100 \%$ & $85 \%$ & $33 \%$ & $62 \%$ & $83 \%$ & $62 \%$ & $67 \%$ & $85 \%$ \\
\hline Hippocampus & $67 \%$ & $85 \%$ & 0 & $8 \%$ & $33 \%$ & $46 \%$ & 0 & $38 \%$ \\
\hline Locus coeruleus & $83 \%$ & $92 \%$ & 0 & 0 & $17 \%$ & $62 \%$ & 0 & $8 \%$ \\
\hline Cerebellum & $83 \%$ & $92 \%$ & 0 & $8 \%$ & $50 \%$ & $62 \%$ & 0 & 0 \\
\hline
\end{tabular}

A

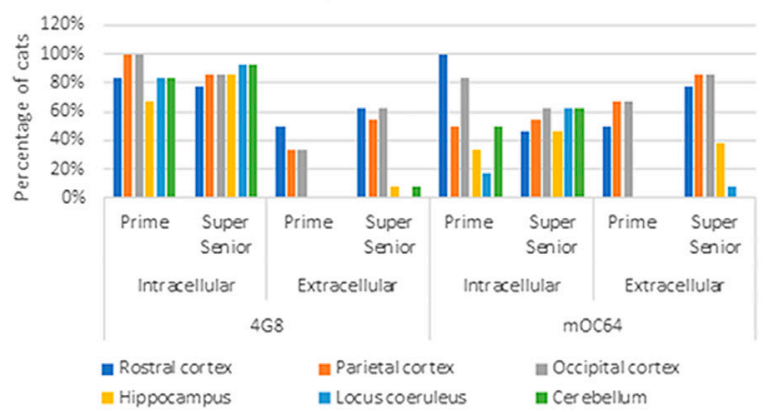

B Intranuclear hyperphosphorylated tau deposits

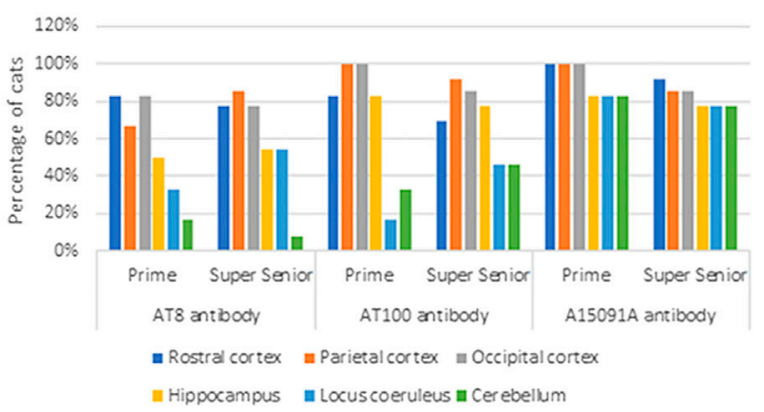

FIGURE 2 |Percentage of cats from the Prime and Super Senior groups showing (A) intracytoplasmic and extracellular A $\beta$ deposits in the different brain regions, with both 4G8 and mOC64 antibodies; and (B) intranuclear labeling of hyperphosphorylated tau (AT8, AT100, and A15091A antibodies) in the different brain regions.

pedigree breed (a Bengal cat). Half of the cats $(52.7 \% ; n=29)$ were females; of these, two thirds $(69 \% ; n=20)$ were neutered, and a third $(31 \% ; n=9)$ were intact. A third of the cats $(30.9 \% ; n=17)$ were males; most of them $(88.3 \% ; n=15)$ were neutered and only $11.7 \%(n=2)$ were intact. The sex of $16.4 \%(n=9)$ of the cats was unknown, mainly because this was not recorded by the pathologists performing the brain extractions. Ages ranged between two and 25 years.

\section{Grouping of Cats}

Since the precise age of some of the cats was uncertain, cats were grouped according to their age as Prime (three to six years old), Mature (seven to 10 years old), Senior (11-14 years old), and Super Senior ( $\geq 15$ years old) (Vogt et al., 2017). After being grouped by age, six cats were in the Prime group; 26 in the Mature group; nine in the Senior group; and 14 in the Super Senior group. A total of ten cats had a confirmed diagnosis of CDS, eight of these cats were in the Super Senior group and two were in the Senior group. (Supplementary material 1).

\section{$\beta$-Amyloid Pathology}

Cats of all ages showed A $\beta$ pathology (i.e., labeling with $4 \mathrm{G} 8$ and mOC64 antibodies). However, elderly cats (i.e., $\geq 15$ years old) had more and larger extracellular $A \beta$ deposits than younger cats. In contrast, younger cats had more positive immunolabelling for intracytoplasmic $A \beta$ (Table 2) (Figure 2A).

Extracellular $A \beta$ deposition was mostly found in the cortex regions. These aggregates had a diffuse pattern (Figure 3A) which, in some cases, formed patches. Congo red and thioflavin-S staining were negative (data not shown). While most of the cats showed a diffuse $A \beta$ distribution, one cat had multiple plaque-like formations (Figure 3B). Both the severity of pathology and the numbers of cats with extracellular $A \beta$ deposits increased with age.

Intraneuronal intracytoplasmic $\mathrm{A} \beta$ immunolabelling was found in the younger cats (i.e., three to six years old; Figure 4) and the percentage of cats with these deposits decreased with age, particularly in cortex. However, the opposite was found in the hippocampus, locus coeruleus, and cerebellum, where the percentage of cats with intracytoplasmic $\mathrm{A} \beta$ immunolabelling increased with age (Figure $\mathbf{2 A}$ ).

One-way ANOVA were performed to determine whether age has an effect on $\mathrm{A} \beta$ pathology (mOC64 antibody) and to assess whether there were differences between the age groups. A statistically significant effect of age on $A \beta$ burden was found in the rostral cortex $\left(\mathrm{F}_{3}=4.55, p=0.005\right)$. Tukey tests 
A

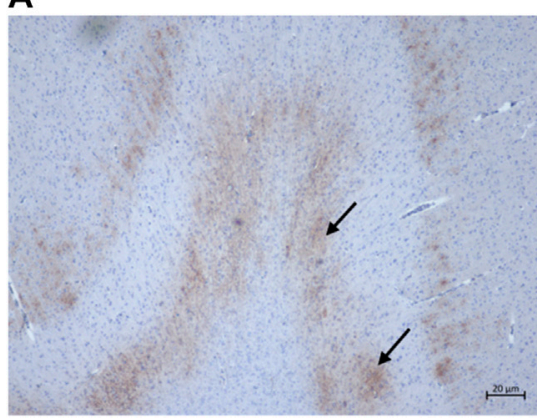

B

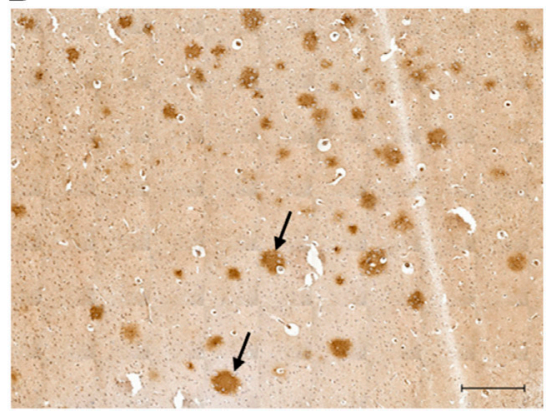

FIGURE 3 |Extracellular B-amyloid (A $\beta$ ) deposition in the cat brain. (A) Diffuse extracellular A $\beta$ deposition in the parietal cortex of a 12-year-old cat (primary antibody mOC64), scale bar $20 \mu \mathrm{m}$; and (B) Extracellular A $\beta$ plaque-like formation in the parietal cortex of a 16-year-old cat (primary antibody mOC64; scale bar $500 \mu \mathrm{m})$.

A

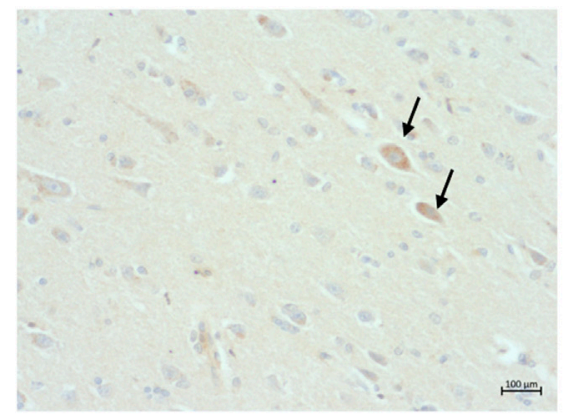

B

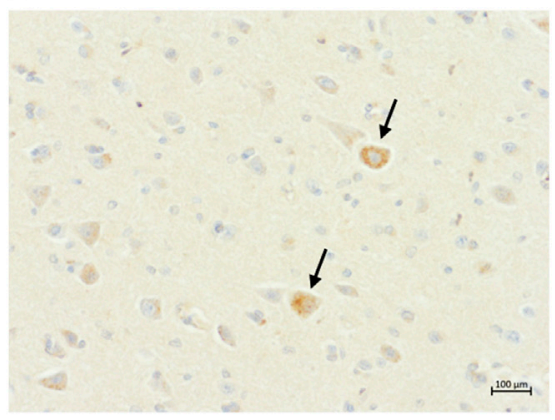

FIGURE 4 | Intraneuronal intracytoplasmic immunolabelling of $B$-amyloid in, (A) the parietal cortex of a 13-year-old cat (primary antibody 4G8); and (B) the occipital cortex of the same 13-year-old cat (primary antibody mOC64), scale bars $100 \mu \mathrm{m}$.

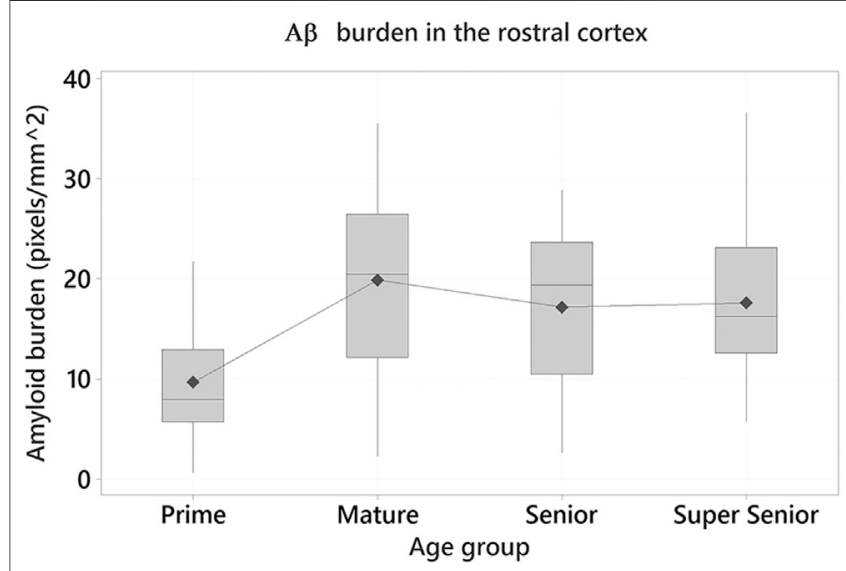

FIGURE 5 |Amyloid- $\beta(A \beta)$ burden in the rostral cortex of the cats in the different age groups. Cats in the Prime group had the lowest $A \beta$ burden when compared to the Mature $(p=0.002)$ and Super Senior groups $(p=0.043)$.

demonstrated the Prime group had the lowest positive immunolabelling, when compared to the other age groups. Statistically significant differences were found between the
Prime and Mature groups (Difference of means $=10.24$; SE of difference $=2.80 ; 95 \%$ CI $[2.94,17.54] ; t=3.66 ; p=0.002)$ and between the Prime and Super Senior groups (Difference of means $=7.93$; $\mathrm{SE}$ of difference $=2.97 ; 95 \% \mathrm{CI}[0.17,15.69] ; t=2.67 ; p=$ 0.043) (Figure 5).

When comparing the $\mathrm{A} \beta$ pathology between the different brain regions, the cerebellum had the lowest load of $\beta$-amyloid when compared to the other regions $\left(\mathrm{F}_{4}=4.58, p=0.001\right)$.

Differences in $A \beta$ pathology between gray and white matter were also assessed. Statistically significant differences in the positive $\mathrm{A} \beta$ labeling were found in all the cortex regions between gray and white matter, with the strongest immunolabelling being present in the gray matter $(p<0.001)$.

To determine whether the extent of $A \beta$ deposits was greater in cats with CDS, we compared the extracellular $A \beta$ load and burden in the different brain regions of cats with CDS with that in agematched controls. However, 2-sample $t$-tests showed no statistically significant differences in the positive $A \beta$ labeling between cats with and without CDS.

\section{Tau Pathology}

Intranuclear and intracytoplasmic immunolabelling of normal tau (i.e., Tau46 antibody) (Figures 6A,B) is found in cats of all 

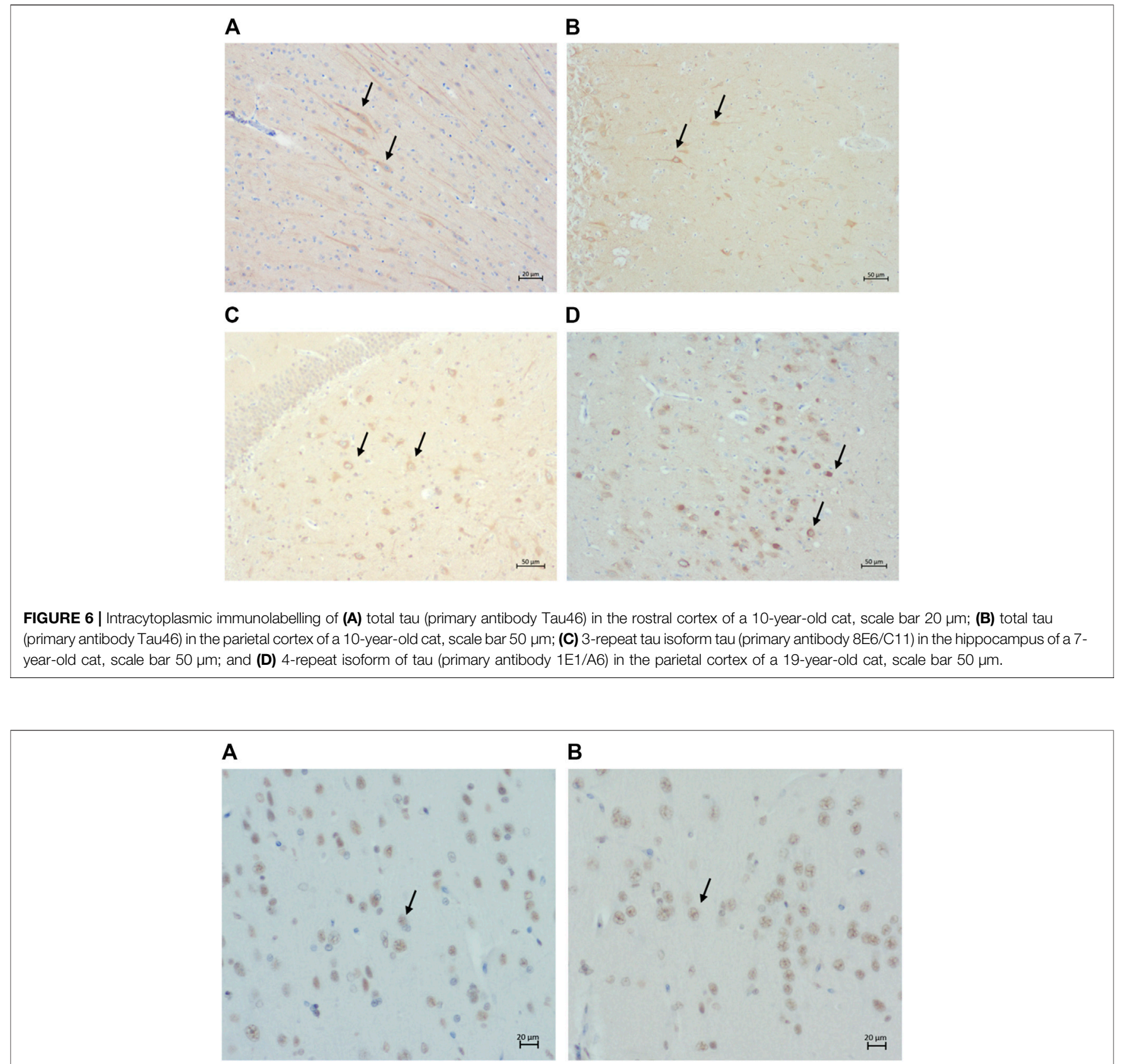

FIGURE 7 | Intranuclear immunolabelling of hyperphosphorylated tau in (A) the parietal cortex of a 14-year-old cat (primary antibody AT8), scale bar $20 \mu \mathrm{m}$; and (B) the parietal cortex of a 14-years-old cat (primary antibody AT100), scale bar $20 \mu \mathrm{m}$.

ages and in all brain regions, as was intracytoplasmic immunolabelling of the two isoforms of tau: 3 and 4-repeat (i.e., labelled by $8 \mathrm{E} 6 / \mathrm{C} 11$ and $1 \mathrm{E} 1 / \mathrm{A} 6$ antibodies, respectively) (Figures 6C,D).

Intranuclear and intracytoplasmic immunolabelling for hyperphosphorylated tau (i.e., AT8, AT100, and A15091A antibodies) was also found within neurons. Intranuclear immunolabelling was primarily found in younger cats, whereas intracytoplasmic labelling (i.e., pre-tangles) was found most frequently in elderly cats (i.e., $\geq 15$ years old).
Intranuclear immunolabelling of hyperphosphorylated tau was most commonly found in cortical regions of younger cats (i.e., three to six years old) and the proportion of cats showing positive immunolabelling tended to decrease with age (Figures 7A,B). In contrast, the percentage of cats showing intranuclear immunolabelling tended to increase with age in the locus coeruleus. However, these were not statistically significant (Table 3) (Figure 2B).

Intracytoplasmic immunolabelling for hyperphosphorylated tau (i.e., pre-tangles) was found within neurons with all three 
TABLE 3 | Percentage of cats from the Prime and Super Senior groups showing intranuclear labeling of hyperphosphorylated tau (AT8, AT100, and A15091A antibodies) in neurons in the different brain regions.

\begin{tabular}{|c|c|c|c|c|c|c|}
\hline \multicolumn{7}{|c|}{ Hyperphosphorylated tau } \\
\hline & \multicolumn{2}{|c|}{ AT8 antibody } & \multicolumn{2}{|c|}{ AT100 antibody } & \multicolumn{2}{|c|}{ A15091A antibody } \\
\hline & $\begin{array}{c}\text { Prime } \\
(\%)\end{array}$ & $\begin{array}{c}\text { Super } \\
\text { senior } \\
(\%)\end{array}$ & $\begin{array}{c}\text { Prime } \\
(\%)\end{array}$ & $\begin{array}{c}\text { Super } \\
\text { senior } \\
(\%)\end{array}$ & $\begin{array}{c}\text { Prime } \\
(\%)\end{array}$ & $\begin{array}{c}\text { Super } \\
\text { senior } \\
(\%)\end{array}$ \\
\hline Rostral cortex & 83 & 77 & 83 & 69 & 100 & 92 \\
\hline Parietal cortex & 67 & 85 & 100 & 92 & 100 & 85 \\
\hline Occipital cortex & 83 & 77 & 100 & 85 & 100 & 85 \\
\hline Hippocampus & 50 & 54 & 83 & 77 & 83 & 77 \\
\hline Locus coeruleus & 33 & 54 & 17 & 46 & 83 & 77 \\
\hline Cerebellum & 17 & 8 & 33 & 46 & 83 & 77 \\
\hline
\end{tabular}

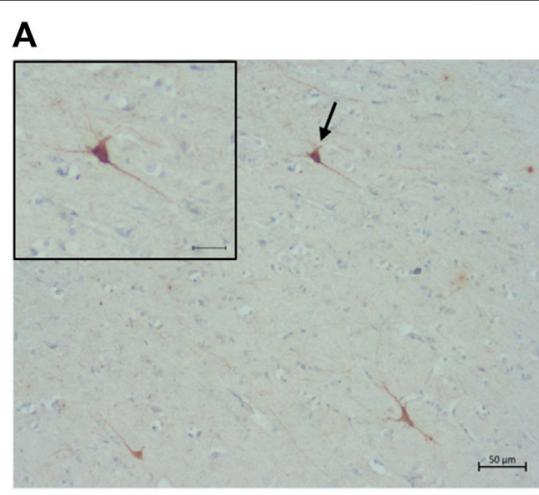

B

FIGURE 8 | Pre-tangles of hyperphosphorylated tau in (A) the rostral cortex of a 10-year-old cat (primary antibody AT8), scale bar 50 $\mu$ m; and (B) the occipital cortex of a 19-year-old (primary antibodyAT100), scale bar $100 \mu \mathrm{m}$.

antibodies and its presence was confirmed by Silver-Gallyas staining (data not shown).

A total of 14 cats were shown to have pre-tangles with the AT8 antibody (Figure 8A). Of these, one was in the Prime group, two in the Mature group, one in the Senior group, and ten in the Super Senior group. Similarly, 15 cats were shown to have pre-tangles with the AT100 antibody (Figure 8B), 14 of which also showed positive staining with AT8. The cat that showed labelling of pre-tangles with AT100 only was in the Prime group. Of note, the cats that had pre-tangles showed little to no intranuclear tau immunolabelling.

Six out of ten cats with a confirmed diagnosis of CDS had pretangles labelled by both AT8 and AT100 mostly in the cerebral cortex, and an additional cat with CDS had a similar distribution of pretangles that were labelled by AT100 only. In contrast, labeling of pretangles with the A15091A antibody, which binds to phosphorylated Ser 262 in human tau, was found in only three cats, one in the Mature group, and two in the Super Senior group. One of the positive cats from the Super Senior group had a confirmed diagnosis of CDS, and its pre-tangles were also detected using AT8 and AT100 antibodies.

The proportion of cats in which pre-tangles were detected with both AT8 and AT100 antibodies increased in all of the brain regions in the Super Senior group (Figure 9).

One-way ANOVA showed statistically significant differences in the burden of hyperphosphorylated tau with the AT8 antibody in the hippocampus $\left(F_{3}=3.08, p=0.036\right)$. Tukey tests demonstrated that the Prime group had a higher burden, when compared to other age groups. Statistically significant differences were found between the Prime and Senior groups (Difference of means $=-0.284$; SE of difference $=0.102 ; 95 \% \mathrm{CI}$ [-0.556, -0.012]; $t=-2.77 ; p=0.038$ ) (Figure 10).

Two-sample t-tests showed statistically significant differences in the loads of hyperphosphorylated tau between gray and white matter; higher loads were found in the gray matter of the rostral $(t=2.12, p=0.036)$ and occipital $(t=2.14$, $p=0.035)$ cortices, with AT8 antibody; and in the parietal $(t=$ $2.58, p=0.011)$ and occipital $(t=2.73, p=0.007)$ cortices, with AT100 antibody.

The largest numbers of pre-tangles were found in cats with CDS. Most of these cats $(6 / 10)$ were shown to have pre-tangles with both the AT8 and AT100 antibodies in the rostral cortex $\left(\mathrm{X}^{2}\right.$ $=31.534, p<0.001$; and $\mathrm{X}^{2}=35.224, p<0.001$, respectively), the parietal cortex $\left(X^{2}=11.6, p=0.001\right.$; and $X^{2}=19.512, p<0.001$, respectively), the occipital cortex $\left(\mathrm{X}^{2}=22.25, p<0.001\right.$; and $\mathrm{X}^{2}=$ $32.245, p<0.001$, respectively), and the hippocampus $\left(\mathrm{X}^{2}=8.709\right.$, $p=0.003$; and $\mathrm{X}^{2}=11.583, p=0.001$, respectively) when compared with age-matched controls.

It could be argued that the intranuclear hyperphosphorylated tau may be a consequence of nonspecific binding of the antibody 

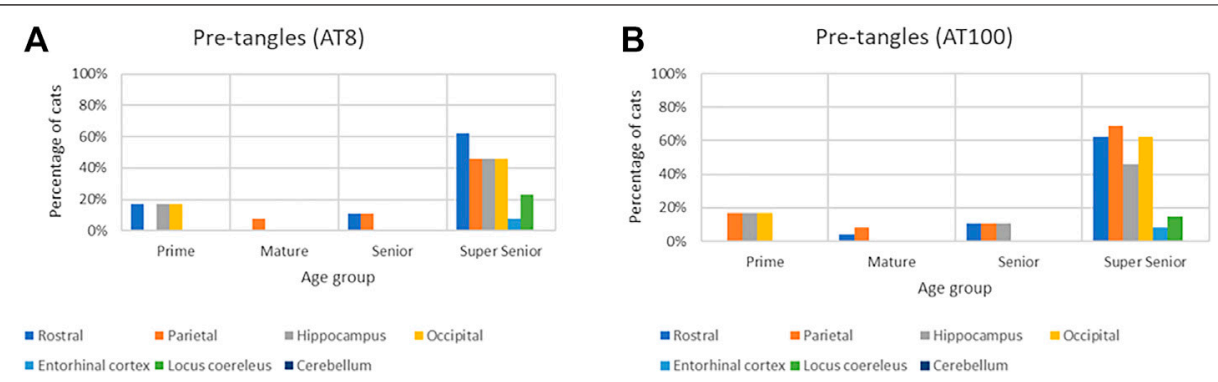

FIGURE 9 | Association of the presence of pre-tangles with age in different brain regions with both AT8 (A) and AT100 (B) antibodies.

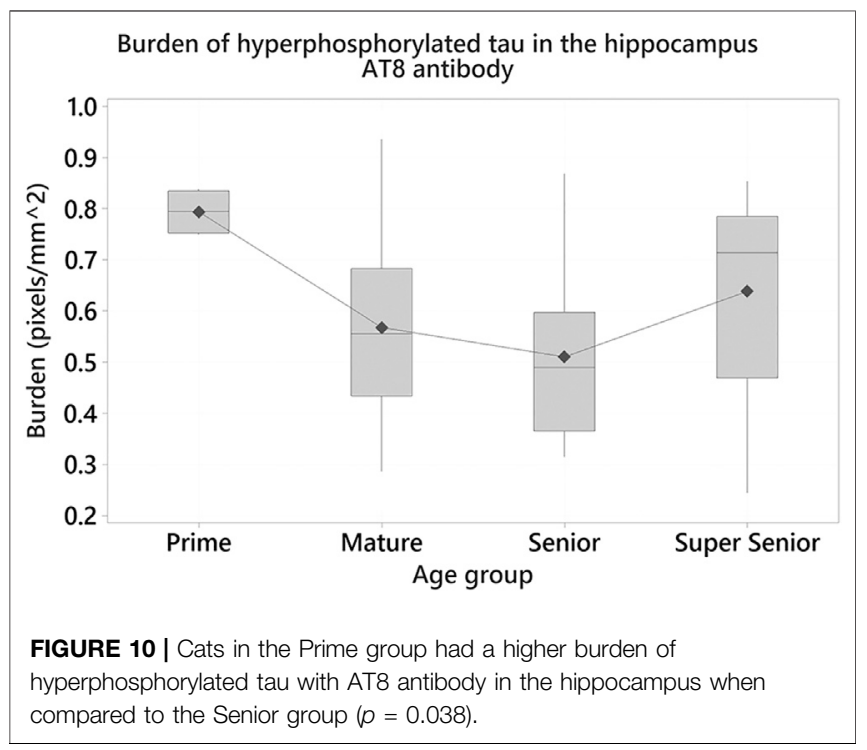

to normal tau, hence, a number of controls were included. To confirm that intranuclear immunolabelling was not caused by artifact or non-specific staining, IHC was performed using antibodies with irrelevant specificities but the same isotypes as AT8, AT100, and A15091A antibodies as negative controls (Figures 11A,B). To validate that the intranuclear labelling was detecting hyperphosphorylated tau, it was shown that in tissue sections dephosphorylated using alkaline phosphatase, intranuclear immunolabelling of tau was abolished (Figures 11C,D).

Finally, a Western blot of different subcellular fractions extracted from a frozen cat brain (a 10-year-old cat) confirmed the presence of intranuclear hyperphosphorylated tau in both the soluble nuclear fraction and the chromatin bound fraction using the AT8 antibody, with a higher level of expression in the soluble nuclear fraction. Hyperphosphorylated tau was also found in the cytoplasm; however, it was not detected in the cytoskeletal fraction (Figure 12). A Western blot using an antibody against total tau (Tau46) gave similar results, although tau was also found in the cytoskeletal fraction with this antibody (data not shown).

\section{DISCUSSION}

This study shows that elderly cats develop both $A \beta$ and tau pathologies; with large extracellular $A \beta$ deposits and the presence of intracytoplasmic pre-tangles of hyperphosphorylated tau within neurons. Of note, the largest numbers of pre-tangles were found in the brains of cats with CDS. In contrast, younger cats generally had more intracytoplasmic $A \beta$ deposits within neurons, and no pre-tangles; however, they did have higher intranuclear tau labelling, unlike the older cats.

\section{$A \beta$ Pathology in the Cat Brain and Its Similarities to AD}

Extracellular deposits of $A \beta$ were found in the brains of most of the cats in this study. While there was some variability between the individual brains, which also occurs in humans (Braak and Braak, 1991; Thal et al., 2002), a similar distribution pattern was observed in most of them (see below).

Cats of all ages had extracellular $A \beta$ deposits; however, the extent of these deposits varied between age groups. Smaller deposits were found in younger cats, whereas the largest deposits were found in cats of the Mature and Super Senior group, particularly in the rostral cortex. Despite cats in the Prime group having smaller $A \beta$ deposits than cats in the Senior group, this was not statistically significant. While this could be due to the lack of power, it is also possible that $A \beta$ accumulation suddenly increases, reaching high levels when cats are between $7-10$ years old (Mature group) and then slowly progresses over time.

In the current study, the youngest cat with extracellular $A \beta$ deposits was four years old; this contradicts previous studies that only reported $A \beta$ deposits in cats over eight years of age (Cummings et al., 1996; Head et al., 2005; Gunn-Moore et al., 2006; Chambers et al., 2015). Extracellular A $\beta$ deposition in cats in the Prime group was only found in the cortical areas (mainly rostral cortex with 4G8 labelling, and parietal and occipital cortices with mOC64). That $A \beta$ deposits start accumulating in cortical regions in younger cat brains is perhaps not surprising. According to Braak and Braak, these are the same regions where $A \beta$ deposition starts in the human brain (i.e., Stage I) (Braak and Braak, 1991; Thal et al., 2002). In humans, $A \beta$ can begin accumulating in individuals as young as 20 -years-old, based upon in vivo PET imaging, which is decades before developing any type of cognitive decline or dementia (Gonneaud et al., 2017). According to International Cat Care and their equivalence table between human and cat ages, a cat of four years old correspond to a human of $\sim 32$ years old (Vogt et al., 2017), so this correlates well with when $A \beta$ starts accumulating in humans. 
A

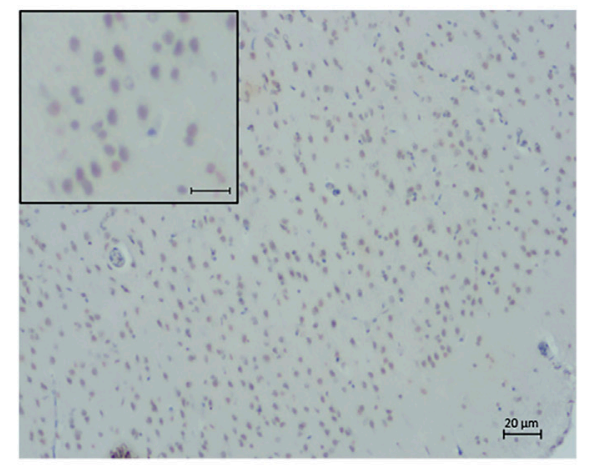

C

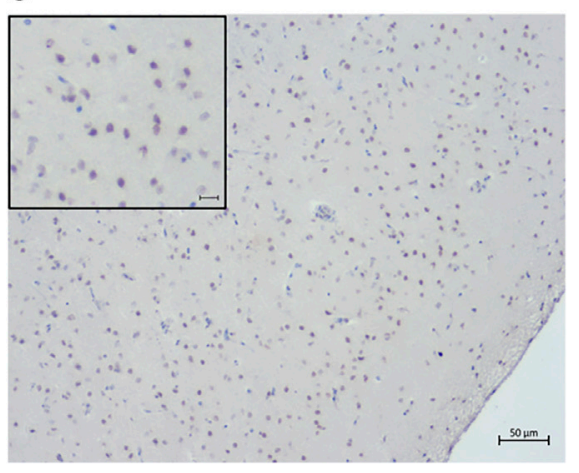

B

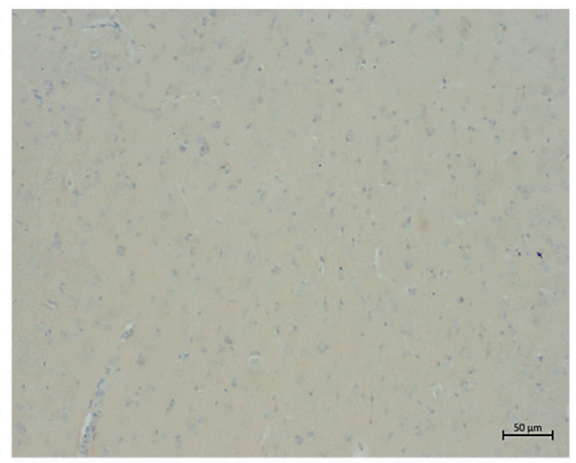

D

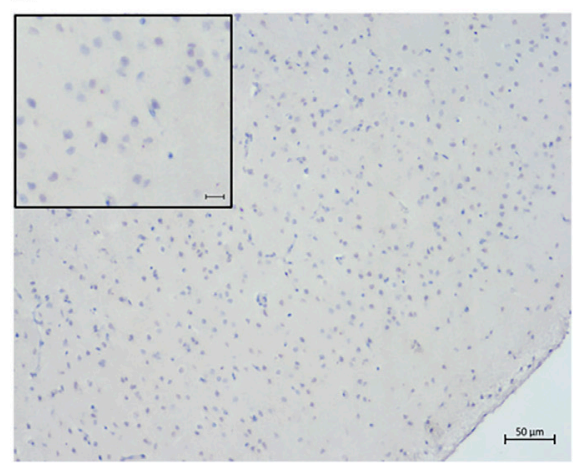

FIGURE 11 | Confirmation of the specificity of intranuclear immunolabeling for hyperphosphorylated tau. Sections of rostral cerebral cortex from a 14-year-old cat were labeled by IHC using either AT8 (A) or an isotype control (B) as primary antibody. Intranuclear staining was not seen in the isotype control section (B). Sections of rostral cerebral cortex from an 18-year-old cat were labeled with AT8 either without (C) or following alkaline phosphatase (AP) treatment (D). No positive intranuclear staining was seen in the AP treated section (D). Scale bars $=20 \mu \mathrm{m}$ in (A) and $50 \mu \mathrm{m}$ in (B), (C), and (D).

Extracellular A $\beta$ deposits were only found in the hippocampus in cats in the Super Senior group. Since cats in this age group also had larger deposits of $\mathrm{A} \beta$ in all the cortical brain regions, it is perhaps logical to conclude that, after accumulating in the cortical areas, $A \beta$ pathology in cats progresses to the hippocampus. This same pattern has been described in the human brain during Stage II of A $\beta$ progression (Braak and Braak, 1991; Thal et al., 2002). However, more studies are needed to confirm this pattern of progression in cats.

While the cortical regions and the hippocampus were the regions most affected by extracellular $A \beta$ deposition in cats, the cerebellum was least affected. In humans, the cerebellum is only affected during the final stage of $A \beta$ progression (i.e., Stage V) (Braak and Braak, 1991; Thal et al., 2002). Hence, it is possible that these cats did not live long enough to allow progression of $A \beta$ deposition to the cerebellum.

Larger extracellular $A \beta$ deposits were found affecting gray matter, when compared to white matter; this was the same in all cortical regions. This also correlates to the $A \beta$ progression proposed by Braak and Braak, as gray matter is initially affected during Stage I, progressing to the white matter during Stage II of disease (Braak and Braak, 1991).

As has been shown previously, extracellular $A \beta$ deposits in cats have a diffuse pattern (Head et al., 2005; Gunn-Moore et al., 2006;
Chambers et al., 2015). This may explain why they were not detected with Congo red or thioflavin-S staining. However, in some cases, these diffuse deposits did form denser patches. This distribution pattern, including the occasional formation of patches, has been previously documented in the cat brain (Head et al., 2005; Gunn-Moore et al., 2006; Chambers et al., 2015). Of note, these deposits only contain the A $\beta 42$ peptide (Cummings et al., 1996; Head et al., 2005).

Diffuse $A \beta$ deposits in humans are mainly formed from this same peptide $(\mathrm{A} \beta 42)$; these are believed to be an early stage of plaques (Hardy and Allsop, 1991; Perl, 2010). Interestingly, one 16-year-old cat in the current study had accumulations of $A \beta$ in well-defined plaque-like structures. This may be a sporadic occurrence, which is supported by finding that the oldest cat in this study (a 25-year-old-cat) had diffuse $\mathrm{A} \beta$ deposition but no plaque-like structures. However, it is also possible that while some cats can form more compact senile plaques, few of them live long enough to do so. Further studies are required to confirm this hypothesis.

No statistically significant difference was found in the presence and/or extent of $\mathrm{A} \beta$ pathology between cats with and without CDS. This may be due to the low numbers of cats with a confirmed diagnosis of CDS. It is also plausible that $A \beta$ pathology in cats does not correlate to the behavioral changes 


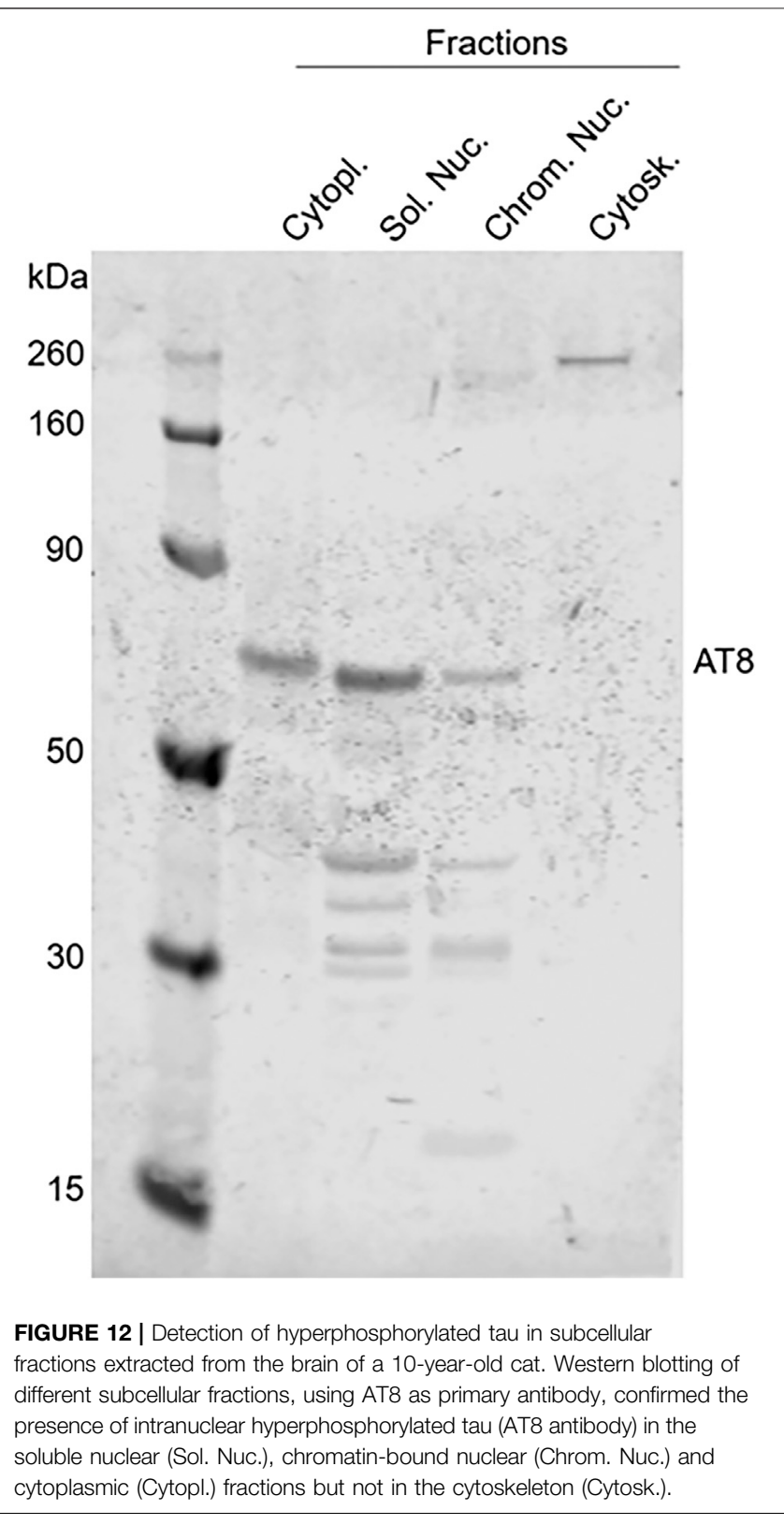

related to CDS, as previously suggested (Head et al., 2005). In humans, there is still contradictory evidence regarding the correlation between $A \beta$ pathology and cognitive impairment. It is true that some studies have found a correlation between $\mathrm{A} \beta$ deposition and cognitive decline (Blessed et al., 1968). However, these studies have some caveats; for instance, they used silver stains that do not differentiate between senile plaques and diffuse deposits. In contrast, there is mounting evidence showing that $\mathrm{A} \beta$ pathology does not correlate with cognitive decline; moreover, there have been individuals with significant $\mathrm{A} \beta$ accumulation and no signs of cognitive impairment (Braak and Braak, 1996; Aizenstein et al., 2008; Villemagne et al., 2011; Ricciarelli and Fedele, 2017).

In humans, $\mathrm{A} \beta$ deposits accumulate within the cytoplasm of cells, particularly neurons, as well as in the extracellular spaces.
Intracytoplasmic $\mathrm{A} \beta$ deposition is believed to begin at a young age and to progress with age (Gouras et al., 2000; Baker-Nigh et al., 2015). For this reason, it has been suggested that intracellular $\mathrm{A} \beta$ has a normal function, potentially as an antioxidant (Smith et al., 2002; Nunomura et al., 2010). During early stages, intracytoplasmic $A \beta$ is expressed at low concentrations and does not produce significant damage (Volloch et al., 2020); however, as individuals get older, it continues to accumulate, until it reaches higher concentrations and becomes toxic (Puzzo and Arancio, 2013). High concentrations of intracytoplasmic $A \beta$ are linked to mitochondrial and cytoskeleton dysfunction, plus synaptic alterations (Gouras et al., 2000; LaFerla et al., 2007; Volloch et al., 2020). Furthermore, once a neuron dies, $A \beta$ is released into the extracellular space, where it may promote further $A \beta$ accumulation (LaFerla et al., 2007). Not surprisingly, correlations between the amount of intra and extracellular $A \beta$ have been reported, as intracellular $A \beta$ concentrations tend to decrease when extracellular deposits accumulate (Mori et al., 2002; Baker-Nigh et al., 2015).

In the present study, cats of all ages show evidence of intracytoplasmic $A \beta$ deposition, predominantly within neurons in cortical areas. When assessing these areas, the proportion of cats with intracellular immunolabelling was highest in the Prime group, and it tended to decrease with age, especially where large extracellular $A \beta$ deposits were found. However, in other areas, such as the hippocampus, locus coeruleus, and cerebellum, the proportion of cats with intracytoplasmic $A \beta$ labelling increased with age. It could be argued that this positive intracytoplasmic labelling may represent APP, rather than $A \beta$, as the $4 \mathrm{G} 8$ antibody binds to the residues 17-24 of $\mathrm{A} \beta$, plus APP; however, intracytoplasmic labelling was also found with the mOC64 antibody, which is a conformation-specific antibody that recognizes monomers, oligomers, and fibrils of $\mathrm{A} \beta 42$. Furthermore, thioflavin-S staining confirmed the amyloid structure. As is the case with humans (as discussed above), these findings suggest that intracytoplasmic $A \beta$ starts accumulating in cortical areas of the cat brain at a young age, but then decreases in these regions as cats get older and extracellular $A \beta$ accumulation increases. Longitudinal studies are needed to determine the progression of these deposits both within the cells and in the extracellular spaces, in addition to looking at the correlation between them. However, given the current lack of a suitable in vivo methods of doing this, it may be possible to assess $A \beta$ in the cerebrospinal fluid of cats to determine the progression of $A \beta$ pathology, which could be later correlated with the neuropathology.

\section{Tau Pathology in the Cat Brain and Its Similarities to AD}

The current study found that non-phosphorylated tau protein was present in the brains of cats of all ages using all three tau antibodies. One of the antibodies, Tau46, binds to the C-terminus of the protein, while the other two $(8 \mathrm{E} 6 / \mathrm{C} 11$ and $1 \mathrm{E} 1 / \mathrm{A} 6)$ bind to the three and four repeats isoforms of the protein, respectively. Labelling with these three antibodies showed that tau was highly 
expressed in all of the brain regions, in all of the cats at all ages, as expected. This correlates with the expression of this protein and its isoforms in the adult human brain (Goedert et al., 1989; Šimić et al., 2016).

Hyperphosphorylated tau was detected within the cytoplasm of neurons in some of the cats, using all three antibodies against hyperphosphorylated tau (i.e., AT8, AT100, and A15091A). Since these aggregates tended to occupy the whole cytoplasm and yet appeared in neurons with normal morphology, they are believed to be pre-tangles (Braak and Del Tredici, 2010), which have been reported previously in cat brains (Head et al., 2005; Gunn-Moore et al., 2006; Chambers et al., 2015).

Pre-tangles were primarily observed in the cortex of cats in the Super Senior group, suggesting that significant tau pathology starts in cortical regions of the cat brain. If true, this may represent a difference in the onset of tau pathology between cats and humans, as it is known to start in the transentorhinal cortex in the latter (Braak and Braak, 1991). However, since the transentorhinal cortex was not assessed in the present study, it is impossible to determine whether tau pathology truly begins in the cortical regions or in the transentorhinal cortex in this species. In addition to the cortex, one of the cats in this study, diagnosed with CDS had small numbers of pre-tangles within their entorhinal cortex.

The load of pre-tangles varied between gray and white matter. The largest loads of pre-tangles were noted in the rostral and occipital cortices in gray matter.

Positive immunolabelling for hyperphosphorylated tau was also found in the hippocampus, where statistically significant differences were found in the burden between the Prime and Senior groups, with a higher burden seen in the Prime group, which was unexpected. However, we noted that the ImageScope image analysis software cannot distinguish nuclear from cytoplasmic labeling. If this is true, it is possible that the positive staining seen in the Prime group corresponds to intranuclear staining, rather than pre-tangles; after visual inspection of the slides this was found to be the case, with the intranuclear staining being stronger in the cats in the Prime group.

Interestingly, few pre-tangles were found in the cortex of two cats in the Prime group (i.e., one 4-year-old cat and one 6-yearold cat). Of note, this 4-year-old cat was the same cat that had extracellular deposition of $A \beta$. While finding pre-tangles in such young cats was unexpected, it was not entirely surprising. It is possible that these cats had undiagnosed neurological disorder that produced tauopathy, or that their age was miscalculated (which is not uncommon in cats that come from a rescue shelter), and they were in fact older than recorded. However, it is also plausible that tau pathology starts at a young age in some cats. Braak suggested that the early stages of tau pathology may occur in younger individuals, as NFT have been found in people under 25 years of age (Braak and Del Tredici, 2010); however, as individuals get older, the number of NFT increases, leading to more severe stages of disease (Braak and Del Tredici, 2010).

While no statistically significant differences were found in either the load or the burden of pre-tangles between cats with and without CDS, associations were found between the presence of pre-tangles and CDS; this is nuanced, but important. Finding an association between the presence of pre-tangles and CDS is not surprising; half of the cats shown to have pre-tangles had a confirmed diagnosis of CDS. This was supported by Chisquare tests that showed that cats with CDS had larger numbers of pre-tangles, compared to cats without CDS. In humans, a strong association has been reported between the presence of NFT and cognitive impairment (Nelson et al., 2012). Furthermore, the progression stages of tau pathology have been associated with different stages of cognitive impairment and dementia. While cognition is known to remain unaffected during Stages I and II, a mild cognitive impairment has been associated to Stages III and IV and, finally, $\mathrm{AD}$ is associated with the most severe stages of pathology (i.e., Stages V and VI) (Braak and Del Tredici, 2010).

While there is a plethora of evidence describing the involvement of NFT in the development of neurodegeneration and $\mathrm{AD}$, recent studies have shown that these insoluble tau aggregates are not as toxic as it was originally proposed (Mi and Johnson, 2006; Mroczko et al., 2019). Instead, it has been suggested that either monomers or soluble oligomers with abnormal post-translational modifications, such as acetylation and increased phosphorylation at specific residues (e.g., acetylation of lysines K174 and K281; and phosphorylation of Thr231 and Tyr18) play a vital role in causing neuronal dysfunction (Irwin et al., 2012; Tracy and Gan, 2017; Neddens et al., 2018; Guha et al., 2020). The role of tau in the development of $\mathrm{AD}$ and cognitive decline remains unclear. On one hand, tau is believed to be the cause and potential trigger of disease, on the other, more recent evidence proposes that it plays a protective role instead (see below).

The presence of intranuclear was first reported in the early nineties in human neuroblastoma (Loomis et al., 1990; Greenwood and Johnson, 1995) and other cells, such as fibroblasts and lymphocytes (Thurston et al., 1996). Of note, one of these studies found that around $16 \%$ of the total tau protein is located within the nucleus, particularly in the chromatin (Greenwood and Johnson, 1995).

In the present study, in addition to IHC labelling of intranuclear tau, Western blots demonstrated that both hyperphosphorylated and non-phosphorylated states of tau were present in the nuclear fraction of cat brain extracts. In humans, both states of intranuclear tau are also known to exist and are believed to have different functions (Greenwood and Johnson, 1995; Rady et al., 1995).

Studies in rodents have shown that nuclear nonphosphorylated tau binds and protects DNA after induced oxidative stress or heat-shock (Papasozomenos and Su, 1991; Sultan et al., 2011; Maina et al., 2016). These two processes have been shown to promote the translocation and accumulation of non-phosphorylated tau into the nucleus and nucleolus in order to protect and maintain the integrity of DNA (Papasozomenos and Su, 1991; Sultan et al., 2011). These studies have shown that there is a significant dephosphorylation of cytoplasmic tau and an increase in nuclear tau immediately after inducing stress; whereas the opposite was observed after $24 \mathrm{~h}$, with an increase in cytoplasmic tau phosphorylation and a decrease in nuclear tau (Sultan et al., 2011). Because of this, it has been suggested that phosphorylation may regulate the translocation of tau between the nucleus and the cytoplasm (Sultan et al., 2011). Furthermore, abnormal phosphorylation may alter the ability of tau to translocate and/or may affect its ability to bind to DNA and 
protect it from damage (Lefebvre et al., 2003; Sultan et al., 2011; Qi et al., 2015; Ulrich et al., 2018).

Nuclear tau is reported in the brains of AD patients, however, its role in the development of disease remains unclear (Maina et al., 2016). Following the amyloid cascade hypothesis, $A \beta$ deposits may promote abnormal phosphorylation of tau, altering its translocation to the nucleus, and impeding its binding and protection of DNA (Maina et al., 2016), as described above.

In the present study, hyperphosphorylated intranuclear tau was primarily found within the cortical neurons of the cats. However, while no statistically significant differences were found between the different age groups, cats in the Prime group tended to have more hyperphosphorylated intranuclear tau, compared to the older age groups. The opposite was seen with in the cats that had pre-tangles, which had little or no intranuclear tau. In contrast, studies in human brains show that nuclear tau increases with age, reaching the highest levels at geriatric stages (Gil et al., 2017); however, in AD, intranuclear tau significantly decreases in the hippocampus and cortex, completely disappearing in advanced stages of disease (Hernández-Ortega et al., 2016).

There are a number of limitations to the present study. Since the neuropathology was only assessed post-mortem, the findings showed in this study represent a snapshot of the neuropathology of aging and CDS in cats. Further studies are needed to determine at what age these changes begin to occur and how the disease progresses over time. Furthermore, since neither behavioral changes nor cognition were formally assessed in most of the cats, no correlations can be evaluated between these and the presence and/or severity of the neuropathology.

Another limitation arose when trying to trace and retrieve the cats' information, particularly where brains were collected several years before the study started; no information was recorded for some of the cats (i.e., sex, exact age, or medical record). Having this information would have helped to understand and/or explain the neuropathological changes observed in these cats, especially in the younger ones.

A limitation concerning the sectioning of the brains was also identified. All brains were manually sectioned by one of the authors (LS) without using a brain slicer, as had been developed for rats' and mice' brains. This resulted in slight variations in the thickness of the brain slices. In addition, there were natural individual variation in both the size and the shape of the brains. Even though anatomical landmarks were used to minimize variation when slicing the brains, there were differences in the size of some of the structures such as the hippocampus and cerebellum. This impeded analyzing these regions in more detail; for example, since the hippocampus was not sectioned in the exact same level for all the cats, assessing differences between the regions within the hippocampus could not be assessed.

Finally, the inability of the Positive Pixel algorithm to differentiate between nuclear or cytoplasmic immunolabelling represents a limitation. Since all the slides were visually inspected before being scanned, this issue was identified promptly. As a result of this limitation, and to examine the nuclear immunolabelling in more detail, the Nuclear algorithm was used in addition to the Positive Pixel quantification.
In summary, and despite the limitations noted above, the current study is the largest yet to examine the brains of cats of various ages for the presence or absence of $A \beta$ and hyperphosphorylated tau deposits. This study found that cats accumulate $A \beta$ as intracytoplasmic deposits and diffuse extracellular deposits; in addition, we found hyperphosphorylated tau within neurons, either as intranuclear or intracytoplasmic deposits, with the cytoplasm representing pretangles. The extent of extracellular $A \beta$ deposits and the number of pre-tangles increase with age, as does the percentage of cats with pretangles. Finally, the presence and progression of these pathologies in terms of their brain location are similar to those seen in humans with $\mathrm{AD}$, suggesting that the cat has the potential to be a natural model for the study of this condition.

\section{DATA AVAILABILITY STATEMENT}

The raw data supporting the conclusions of this article will be made available by the authors, without undue reservation.

\section{ETHICS STATEMENT}

The animal study was reviewed and approved by The Veterinary Ethical Review Committee from The Royal (Dick) School of Veterinary Studies, The University of Edinburgh. Written informed consent was obtained from the owners for the participation of their animals in this study.

\section{AUTHOR CONTRIBUTIONS}

The present study was designed by LS, EFH, and DG-M. The laboratory work, collection of data and statistical analyses were performed by LS. The section on the quantification of proteins was performed by $\mathrm{LS}, \mathrm{AM}$, and $\mathrm{EH}$. The initial draft of the present manuscript was produced by LS, EFH, and DG-M with later editing by $\mathrm{AM}$ and $\mathrm{EH}$.

\section{ACKNOWLEDGMENTS}

The authors would like to thank The National Council of Science and Technology for funding LS PhD studies, and Declan King, Rona Barron, Chandra Logie, and Neil McIntyre for their help with the collection of brains and processing them in the laboratory. The authors would also like to thank The Soulsby Foundation and Alzheimer's Research United Kingdom (ARUK) for funding LS visit to The Department of Pathology and Laboratory Medicine at The University of California, Irvine.

\section{SUPPLEMENTARY MATERIAL}

The Supplementary Material for this article can be found online at: https://www.frontiersin.org/articles/10.3389/fragi.2021.684607/ full\#supplementary-material 


\section{REFERENCES}

Aizenstein, H. J., Nebes, R. D., Saxton, J. A., Price, J. C., Mathis, C. A., Tsopelas, N. D., et al. (2008). Frequent Amyloid Deposition without Significant Cognitive Impairment Among the Elderly. Arch. Neurol. 65 (11), 1509-1517. doi:10.1001/ archneur.65.11.1509

Baker-Nigh, A., Vahedi, S., Davis, E. G., Weintraub, S., Bigio, E. H., Klein, W. L., et al. (2015). Neuronal Amyloid- $\beta$ Accumulation within Cholinergic Basal Forebrain in Ageing and Alzheimer's Disease. Brain 138 (6), 1722-1737. doi:10. 1093/brain/awv024

Blessed, G., Tomlinson, B. E., and Roth, M. (1968). The Association between Quantitative Measures of Dementia and of Senile Change in the Cerebral Grey Matter of Elderly Subjects. Br. J. Psychiatry 114 (512), 797-811. doi:10.1192/bjp. 114.512 .797

Braak, H., and Braak, E. (1991). Neuropathological Stageing of Alzheimer-Related Changes. Acta Neuropathol. 82 (4), 239-259. doi:10.1007/bf00308809

Braak, H., and Braak, E. (1996). Evolution of the Neuropathology of Alzheimer's Disease. Acta Neurol. Scand. 94 (S165), 3-12. doi:10.1111/j.1600-0404.1996. tb05866.x

Braak, H., and Del Tredici, K. (2010). "Neurofibrillary Tangles," in Encyclopedia of Movement Disorders (Amsterdam, Netherlands: HP), Vol. 2, 265-269. doi:10. 1016/b978-0-12-374105-9.00269-0

Brellou, G., Vlemmas, I., Lekkas, S., and Papaioannou, N. (2005). Immunohistochemical Investigation of Amyloid Beta-Protein (Abeta) in the Brain of Aged Cats. Histol. Histopathol. 20 (3), 725-731. doi:10.14670/HH20.725

Chambers, J. K., Tokuda, T., Uchida, K., Ishii, R., Tatebe, H., Takahashi, E., et al. (2015). The Domestic Cat as a Natural Animal Model of Alzheimer's Disease. Acta neuropathol. Commun. 3, 78. doi:10.1186/s40478-015-0258-3

Colle, M., Hauw, J. J., Crespeau, F., Uchihara, T., Akiyama, H., Checler, F., et al. (2000). Vascular and Parenchymal A $\hat{I}^{2}$ Deposition in the Aging Dog: Correlation with Behavior. Neurobiol. Aging 21 (5), 695-704. doi:10.1016/ s0197-4580(00)00113-5

Cummings, B., Satou, T., Head, E., Milgram, N. W., Cole, G. M., Savage, M. J., et al. (1996). Diffuse Plaques Contain C-Terminal A $\beta 42$ and Not A $\beta 40$ : Evidence from Cats and Dogs. Neurobiol. Aging 17 (4), 653-659. doi:10.1016/s01974580(96)00062-0

Gil, L., Federico, C., Pinedo, F., Bruno, F., Rebolledo, A. B., Montoya, J. J., et al. (2017). Aging Dependent Effect of Nuclear Tau. Brain Res. 1677, 129-137. doi:10.1016/j.brainres.2017.09.030

Glabe, C. G. (2006). Common Mechanisms of Amyloid Oligomer Pathogenesis in Degenerative Disease. Neurobiol. Aging 27 (4), 570-575. doi:10.1016/j. neurobiolaging.2005.04.017

Goedert, M., Spillantini, M. G., Jakes, R., Rutherford, D., and Crowther, R. A. (1989). Multiple Isoforms of Human Microtubule-Associated Protein Tau: Sequences and Localization in Neurofibrillary Tangles of Alzheimer's Disease. Neuron 3 (4), 519-526. doi:10.1016/0896-6273(89)90210-9

Gonneaud, J., Arenaza-Urquijo, E. M., Mézenge, F., Landeau, B., Gaubert, M., Bejanin, A., et al. (2017). Increased Florbetapir Binding in the Temporal Neocortex from Age 20 to 60 Years. Neurology 89 (24), 2438-2446. doi:10. 1212/wnl.0000000000004733

Gouras, G. K., Tsai, J., Naslund, J., Vincent, B., Edgar, M., Checler, F., et al. (2000). Intraneuronal A $\beta 42$ Accumulation in Human Brain. Am. J. Pathol. 156 (1), 15-20. doi:10.1016/s0002-9440(10)64700-1

Greenwood, J. A., and Johnson, G. V. W. (1995). Localization and In Situ Phosphorylation State of Nuclear Tau. Exp. Cel. Res. 220 (2), 332-337. doi:10.1006/excr.1995.1323

Guha, S., Fischer, S., Johnson, G. V., and Nehrke, K. (2020). Tauopathy-associated Tau Modifications Selectively Impact Neurodegeneration and Mitophagy in a Novel C. elegans Single-Copy Transgenic Model. Mol. Neurodegeneration 15 (1), 1-16. doi:10.1186/s13024-020-00410-7

Gunn-Moore, D., McVee, J., Bradshaw, J., Pearson, G., Head, E., and Gunnmoore, F. (2006). Ageing Changes in Cat Brains Demonstrated by $\beta$-amyloid and AT8Immunoreactive Phosphorylated Tau Deposits. J. Feline Med. Surg. 8 (4), 234-242. doi:10.1016/j.jfms.2006.01.003
Gunn-Moore, D., Moffat, K., Christie, L.-A., and Head, E. (2007). Cognitive Dysfunction and the Neurobiology of Ageing in Cats. J. Small Anim. Pract. 48 (10), 546-553. doi:10.1111/j.1748-5827.2007.00386.x

Hardy, J., and Allsop, D. (1991). Amyloid Deposition as the central Event in the Aetiology of Alzheimer's Disease. Trends Pharmacol. Sciences 12, 383-388. doi:10.1016/0165-6147(91)90609-v

Head, E., McCleary, R., Hahn, F. F., Milgram, N. W., and Cotman, C. W. (2000). Region-specific Age at Onset of $\beta$-amyloid in Dogs 败. Neurobiol. Aging 21, 89-96. doi:10.1016/s0197-4580(00)00093-2

Head, E., Moffat, K., Das, P., Sarsoza, F., Poon, W. W., Landsberg, G., et al. (2005). $\beta$-Amyloid Deposition and Tau Phosphorylation in Clinically Characterized Aged Cats. Neurobiol. Aging 26 (5), 749-763. doi:10.1016/j.neurobiolaging. 2004.06.015

Hernández-Ortega, K., Garcia-Esparcia, P., Gil, L., Lucas, J. J., and Ferrer, I. (2016). Altered Machinery of Protein Synthesis in Alzheimer's: from the Nucleolus to the Ribosome. Brain Pathol. 26 (5), 593-605. doi:10.1111/bpa.12335

Hyman, B. T., Marzloff, K., and Arriagada, P. V. (1993). The Lack of Accumulation of Senile Plaques or Amyloid Burden in Alzheimer's Disease Suggests a Dynamic Balance between Amyloid Deposition and Resolution. J. Neuropathol. Exp. Neurol. 52, 594-600. doi:10.1097/00005072-19931100000006

Irwin, D. J., Cohen, T. J., Grossman, M., Arnold, S. E., Xie, S. X., Lee, V. M.-Y., et al. (2012). Acetylated Tau, a Novel Pathological Signature in Alzheimer's Disease and Other Tauopathies. Brain 135 (3), 807-818. doi:10.1093/brain/aws013

Jack, C. R., Jr., Shiung, M. M., Weigand, S. D., O’Brien, P. C., Gunter, J. L., Boeve, B. F., et al. (2005). Brain Atrophy Rates Predict Subsequent Clinical Conversion in normal Elderly and Amnestic MCI. Neurology 65, 1227-1231. doi:10.1212/01. wnl.0000180958.22678.91

Kametani, F., and Hasegawa, M. (2018). Reconsideration of Amyloid Hypothesis and Tau Hypothesis in Alzheimer's Disease. Front. Neurosci. 12, 25. doi:10. 3389/fnins.2018.00025

Kraszpulski, M., Soininen, H., Riekkinen Sr., P., and Alafuzoff, I. (1998). Pitfalls in the Quantitative Estimation of $\beta$-amyloid Immunoreactivity in Human Brain Tissue. Histochem. Cel. Biol. 110 (4), 439-445. doi:10.1007/s004180050305

Kuroki, K., Uchida, K., Kiatipattanasakul, W., Nakamura, S.-i., Yamaguchi, R., Nakayama, H., et al. (1997). Immunohistochemical Detection of Tau Protein in Various Non-human Animal Brains. Neuropathology 17, 174-180. doi:10.1111/ j.1440-1789.1997.tb00034.x

LaFerla, F. M., Green, K. N., and Oddo, S. (2007). Intracellular Amyloid- $\beta$ in Alzheimer's Disease. Nat. Rev. Neurosci. 8 (7), 499-509. doi:10.1038/nrn2168

Landsberg, G. M., Denenberg, S., and Araujo, J. A. (2010). Cognitive Dysfunction in Cats: A Syndrome We Used to Dismiss as 'Old Age'. J. Feline Med. Surg. 12, 837-848. doi:10.1016/j.jfms.2010.09.004

Lee, H.-g., Perry, G., Moreira, P. I., Garrett, M. R., Liu, Q., Zhu, X., et al. (2005). Tau Phosphorylation in Alzheimer's Disease: Pathogen or Protector?. Trends Molecular Medicine 11 (4), 164-169. doi:10.1016/j.molmed.2005.02.008

Lefebvre, T., Ferreira, S., Dupont-Wallois, L., Bussière, T., Dupire, M.-J., Delacourte, A., et al. (2003). Evidence of a Balance between Phosphorylation and O-GlcNAc Glycosylation of Tau Proteins-A Role in Nuclear Localization. Biochim. Biophys. Acta (Bba) - Gen. Subjects 1619 (2), 167-176. doi:10.1016/s0304-4165(02)00477-4

Loomis, P. A., Howard, T. H., Castleberry, R. P., and Binder, L. I. (1990). Identification of Nuclear Tau Isoforms in Human Neuroblastoma Cells. Proc. Natl. Acad. Sci. 87 (21), 8422-8426. doi:10.1073/pnas.87.21.8422

Maina, M. B., Al-Hilaly, Y. K., and Serpell, L. C. (2016). Nuclear Tau and its Potential Role in Alzheimer's Disease. Biomolecules 6 (1), 2-20. doi:10.3390/ biom6010009

Mi, K., and Johnson, G. (2006). The Role of Tau Phosphorylation in the Pathogenesis of Alzheimers Disease. Car 3 (5), 449-463. doi:10.2174/ 156720506779025279

Mori, C., Spooner, E. T., Wisniewski, K. E., Wisniewski, T. M., Yamaguchi, H., Saido, T. C., et al. (2002). Intraneuronal A $\beta 42$ Accumulation in Down Syndrome Brain. Amyloid 9 (2), 88-102. doi:10.3109/13506120208995241

Mroczko, B., Groblewska, M., and Litman-Zawadzka, A. (2019). The Role of Protein Misfolding and Tau Oligomers (TauOs) in Alzheimer's Disease (AD). Ijms 20 (19), 4661. doi:10.3390/ijms20194661 
Nakamura, S.-i., Nakayama, H., Kiatipattanasakul, W., Uetsuka, K., Uchida, K., and Goto, N. (1996). Senile Plaques in Very Aged Cats. Acta Neuropathologica 91, 437-439. doi:10.1007/s004010050448

Neddens, J., Temmel, M., Flunkert, S., Kerschbaumer, B., Hoeller, C., Loeffler, T., et al. (2018). Phosphorylation of Different Tau Sites during Progression of Alzheimer's Disease. Acta neuropathol. Commun. 6 (1), 1-15. doi:10.1186/ s40478-018-0557-6

Nelson, P. T., Alafuzoff, I., Bigio, E. H., Bouras, C., Braak, H., Cairns, N. J., et al. (2012). Correlation of Alzheimer Disease Neuropathologic Changes with Cognitive Status: a Review of the Literature. J. Neuropathol. Exp. Neurol. 71 (5), 362-381. doi:10.1097/nen.0b013e31825018f7

Nunomura, A., Tamaoki, T., Tanaka, K., Motohashi, N., Nakamura, M., Hayashi, T., et al. (2010). Intraneuronal Amyloid $\beta$ Accumulation and Oxidative Damage to Nucleic Acids in Alzheimer Disease. Neurobiol. Dis. 37 (3), 731-737. doi:10. 1016/j.nbd.2009.12.012

Papasozomenos, S. C., and Su, Y. (1991). Altered Phosphorylation of Tau Protein in Heat-Shocked Rats and Patients with Alzheimer Disease. Proc. Natl. Acad. Sci. 88 (10), 4543-4547. doi:10.1073/pnas.88.10.4543

Perl, D. P. (2010). Neuropathology of Alzheimer's Disease. Mt Sinai J. Med. 77 (1), 32-42. doi:10.1002/msj.20157

Puzzo, D., and Arancio, O. (2013). Amyloid- $\beta$ Peptide: Dr. Jekyll or Mr. Hyde?. J. Alzheimers Dis. 33 Suppl. 1 (s1), S111-S120. doi:10.3233/JAD-2012-129033

Qi, H., Cantrelle, F.-X., Benhelli-Mokrani, H., Smet-Nocca, C., Buée, L., Lippens, G., et al. (2015). Nuclear Magnetic Resonance Spectroscopy Characterization of Interaction of Tau with DNA and its Regulation by Phosphorylation. Biochemistry 54 (7), 1525-1533. doi:10.1021/bi5014613

Rady, R. M., Zinkowski, R. P., and Binder, L. I. (1995). Presence of Tau in Isolated Nuclei from Human Brain. Neurobiol. Aging 16 (3), 479-486. doi:10.1016/ 0197-4580(95)00023-8

Raz, N. (2004). "The Aging Brain: Structural Changes and Their Implications for Cognitive Aging," in The Ageing Brain: Structural Changes and Their Implications for Cognitive Ageing. Editors R. Dixon, L. Backman, and L. Nilsson (Oxford: Oxford University Press), 115-134. doi:10.1093/acprof: oso/9780198525691.003.0006

Ricciarelli, R., and Fedele, E. (2017). The Amyloid cascade Hypothesis in Alzheimer's Disease: It's Time to Change Our Mind. Curr. Neuropharmacol. 15 (6), 926-935. doi:10.2174/1570159x15666170116143743

Šimić, G., Babić Leko, M., Wray, S., Harrington, C., Delalle, I., Jovanov-Milošević, N., et al. (2016). Tau Protein Hyperphosphorylation and Aggregation in Alzheimer's Disease and Other Tauopathies, and Possible Neuroprotective Strategies. Biomolecules 6 (1), 6. doi:10.3390/biom6010006

Smith, M. A., Casadesus, G., Joseph, J. A., and Perry, G. (2002). Amyloid- $\beta$ and $\tau$ Serve Antioxidant Functions in the Aging and Alzheimer Brain. Free Radic. Biol. Med. 33 (9), 1194-1199. doi:10.1016/s0891-5849(02)01021-3
Sordo, L., and Gunn-Moore, D. (2021). Cognitive Dysfunction in Cats: Update on Neuropathological and Behavioural Changes Plus Clinical Management. Vet. Rec. 188, 1-12.doi:10.1002/vetr.3

Sultan, A., Nesslany, F., Violet, M., Bégard, S., Loyens, A., Talahari, S., et al. (2011). Nuclear Tau, a Key Player in Neuronal DNA Protection. J. Biol. Chem. 286, 4566-4575. doi:10.1074/jbc.m110.199976

Thal, D. R., Rüb, U., Orantes, M., and Braak, H. (2002). Phases of A $\beta$-Deposition in the Human Brain and its Relevance for the Development of AD. Neurology 58 (12), 1791-1800. doi:10.1212/wnl.58.12.1791

Thurston, V. C., Zinkowski, R. P., and Binder, L. I. (1996). Tau as a Nucleolar Protein in Human Nonneural Cells In Vitro and In Vivo. Chromosoma 105 (1), 20-30. doi:10.1007/s004120050155

Tracy, T. E., and Gan, L. (2017). Acetylated Tau in Alzheimer's Disease: An Instigator of Synaptic Dysfunction Underlying Memory Loss. Bioessays 39 (4), 1600224. doi:10.1002/bies.201600224

Ulrich, G., Salvadè, A., Boersema, P., Calì, T., Foglieni, C., Sola, M., et al. (2018). Phosphorylation of Nuclear Tau Is Modulated by Distinct Cellular Pathways. Scientific Rep. 8 (1), 1-14. doi:10.1038/s41598-018-36374-4

Villemagne, V. L., Pike, K. E., Chételat, G., Ellis, K. A., Mulligan, R. S., Bourgeat, P., et al. (2011). Longitudinal Assessment of $A \beta$ and Cognition in Aging and Alzheimer Disease. Ann. Neurol. 69 (1), 181-192. doi:10.1002/ana.22248

Vogt, A. H., Rodan, I., Brown, M., Brown, S., Buffington, C. A. T., Forman, M. J. L., et al. (2017). AAFP-AAHA. J. Feline Med. Surg. 12(1), 43-54. doi:10.1016/j. jfms.2009.12.006

Volloch, V., Olsen, B., and Rits, S. (2020). Alzheimer's Disease Is Driven by Intraneuronally Retained Beta-Amyloid Produced in the AD-specific, $\beta$ APPIndependent Pathway: Current Perspective and Experimental Models for Tomorrow. Ann. Integr. Mol. Med. 2 (1), 90-114. doi:10.33597/aimm.02-1007 Wisniewski, T., Lalowski, M., Bobik, M., Russell, M., Strosznajder, J., and Frangione, B. (1996). Amyloid $\beta$ 1-42 Deposits Do Not lead to Alzheimer's Neuritic Plaques in Aged Dogs. Biochem. J. 313, 575-580. doi:10.1042/ bj3130575

Conflict of Interest: The authors declare that the research was conducted in the absence of any commercial or financial relationships that could be construed as a potential conflict of interest.

Copyright (c) 2021 Sordo, Martini, Houston, Head and Gunn-Moore. This is an open-access article distributed under the terms of the Creative Commons Attribution License (CC BY). The use, distribution or reproduction in other forums is permitted, provided the original author(s) and the copyright owner(s) are credited and that the original publication in this journal is cited, in accordance with accepted academic practice. No use, distribution or reproduction is permitted which does not comply with these terms. 